Review Article

\title{
Understanding short bowel syndrome: Current status and future perspectives
}

\author{
Sara Massironi ${ }^{a, *}$, Federica Cavalcoli $^{b}$, Emanuele Rausa $^{c}$, Pietro Invernizzi $^{\mathrm{d}}$, \\ Marco Braga ${ }^{\mathrm{e}}$, Maurizio Vecchi ${ }^{\mathrm{a}}$ \\ a Gastroenterology and Endoscopy Unit, IRCCS Ca' Granda Foundation, Policlinico Hospital, University of the Study of Milan, Italy \\ b Gastroenterology Unit, National Cancer Institute (INT), Milan, Italy \\ ${ }^{\mathrm{c}}$ Division of Surgical Oncology, ASST Bergamo Ovest, Treviglio, Italy \\ d Division of Gastroenterology and Center for Autoimmune Liver Diseases, San Gerardo Hospital, University of Milano, Bicocca School of Medicine, Monza, \\ Italy \\ e Division of Surgery, San Gerardo Hospital, University of Milano - Bicocca School of Medicine, Monza, Italy
}

\section{A R T I C L E I N F O}

\section{Article history:}

Received 13 August 2019

Accepted 18 November 2019

Available online 28 December 2019

\section{Keywords:}

Home-based parenteral nutrition (HPN)

Intestinal failure

Intestinal transplantation

Malabsorption

Short bowel syndrome (SBS)

Teduglutide

\begin{abstract}
A B S T R A C T
Short bowel syndrome (SBS) is a rare malabsorptive disorder as a result of the loss of bowel mass mostly secondary to surgical resection of the small intestine. Other causes are vascular diseases, neoplasms or inflammatory bowel disease. The spectrum of the disease is widely variable from single micronutrient malabsorption to complete intestinal failure, depending on the remaining length of the small intestine, the anatomical portion of intestine and the function of the remnant bowel. Over the last years, the management of affected patients has remarkably improved with the increase in patients' quality of life and survival, mainly thanks to advances in home-based parenteral nutrition (PN). In the last ten years new treatment strategies have become available together with increasing experience and the encouraging results with new drugs, such as teduglutide, have added a new dimension to the management of SBS.

This review aims to summarize the knowledge available in the current literature on SBS epidemiology, pathophysiology, and its surgical (including intestinal lengthening procedures and intestinal transplantation) and medical management with emphasis on the recent advances.

Moreover, this review attempts to provide the new understanding and recent approaches to SBS complications such as sepsis, catheter thrombosis, and intestinal failure-associated liver disease.
\end{abstract}

(c) 2019 Editrice Gastroenterologica Italiana S.r.l. Published by Elsevier Ltd. All rights reserved.

\section{Introduction}

Short bowel syndrome (SBS) is an uncommon malabsorptive condition most frequently caused by massive surgical resection of the small intestine. SBS is associated with significant morbidity and mortality, reduced quality of life, and high healthcare costs [1]. The clinical manifestations are only partially related with the length of the resected intestine, but it mostly depends on the ability of the residual gastrointestinal tract to compensate the resected part and to adapt functionally to the new anatomical condition. Therefore, the definition of SBS is based upon functional rather than anatomical impairment and it is related to the presence of significant malabsorption of both micronutrients and macronutrients. For

\footnotetext{
* Corresponding author at: Gastroenterology and Endoscopy Unit, Fondazione IRCCS Ca' Granda Ospedale Maggiore Policlinico, Via F. Sforza 35, 20122 Milan, Italy. E-mail addresses: sara.massironi@libero.it, Sara.massironi@unimi.it (S. Massironi).
}

these reasons, the spectrum of the clinical disease is widely variable from only single micronutrient malabsorption to complete intestinal failure, defined as the reduction of the gut function below the minimum necessary for the absorption of macronutrients and/or water and electrolytes. Whether the intestinal function is inadequate to maintain an adequate protein-energy, fluid, electrolytes, or micronutrient balances, intravenous supplementation is required to keep up health and/or growth [2].

Over the last fifty years, the management of these patients has drastically changed: parenteral nutrition (PN) is currently a standard of care for SBS patients, with a wide range of formulations now available for both hospital and outpatient management. However, PN still presents a relevant burden of complications (infective risk, catheter malfunction, thrombosis, and metabolic derangements), healthcare costs and impairment in quality of life [3].

Moreover, recently introduced pharmacological drugs (GLP-2 analogs) have begun to generate a significant impact on the care 
and ultimate management of these patients, giving PN-dependent patients a possibility of reducing $\mathrm{PN}$ requirements.

\section{Epidemiology}

The actual prevalence of SBS in adults worldwide has not been fully clarified mostly because of under-reporting and the absence of reliable patients databases [4,5]. The best estimates are based on numbers of patients receiving long-term parenteral nutrition and/or intravenous fluid support. SBS prevalence has been reported to be 1.4 cases for million in Europe (ranging from 0.4 in Poland to 40 in Denmark) and 30 cases per million in the US [4,6,7]. However, estimates can widely vary between countries. Overall, based on a recent study by Brandt et al. an increase in the worldwide prevalence of SBS has been suggested, indeed the authors reported a more than two-fold increase in the number of patients with SBS over the past 40 years [8].

SBS occurs in about $15 \%$ of adult patients who undergo intestinal resection. Of these up to $75 \%$ of cases result from massive intestinal resection and 25\% from multiple sequential resections [9]. SBS has been more frequently reported in women than men, possibly because of the shorter length of the small intestine in women $v s$. men [10].

For patients with SBS, the rate of PN dependency at 1, 2, and 5 years was reported as $74 \%, 64 \%$, and $48 \%$ and is significantly associated with a remnant small bowel length less than $75 \mathrm{~cm}$ and a large part of the remaining colon [11]. Mortality rates remain high ranging from $30 \%$ to $50 \%$.

\section{Etiology}

Several conditions requiring intestinal resection may lead to SBS [12]. To date, re-surgery performed for complications following a previous abdominal operation is one of the leading causes of SBS in adults and accounts for up to $50 \%$ of patients [13-16]. The cause of re-intervention may be related to intestinal obstruction (mostly secondary to internal hernia or volvulus), ischemia due to vascular injury, or hypotension [12,16].

Mesenteric ischemia remains an important risk factor for SBS [17-19]. Mesenteric vascular disease related to arterial thrombosis or embolism is the most common mechanism of intestinal ischemia [20]. However, ischemia related to drug abuse and coagulation disorders is increasingly reported [21,22]. Non-occlusive mesenteric ischemia is also an important cause of SBS, particularly in critically ill patients.

Neoplasm with or without radiation treatment also accounts for a significant number of SBS cases $[23,24]$. The mechanisms through which a neoplasm may favor SBS are to do with the development of intestinal obstruction, fistulas, perforation, massive resection due to the involvement of mesenteric vessels [25]. Radiotherapy might result in radiation enteritis and the need for resection [23].

Crohn's disease is also a common cause of SBS in adult and accounts for $10 \%-20 \%$ of cases, but its incidence is declining due to less aggressive resective therapy [17,26-28]. Most cases arise in patients with extensive small intestinal or ileocolonic involvement and of penetrating type [29].

Finally, resection for trauma or some benign conditions, such as intestinal pseudo-obstruction, or recurrent bleeding results in SBS in approximately $10 \%$ of the cases $[12,30]$ (refer to Supplementary file).

\section{Pathophysiology}

Several factors determine the severity and type of malabsorption in SBS. The primary pathophysiological mechanism of malabsorption is the loss of intestinal absorptive surface secondary to small bowel surgical removal or loss of function. In addition, the loss of different anatomical sites may result in specific nutrients deficiency. Terminal ileum lack is responsible for malabsorption of vitamin $B_{12}$, fat-soluble vitamins, and bile acids [31]. The loss of the distal ileum and ileocecal valve causes the lack of inhibitory hormones signals that result in intestinal transit acceleration, gastric hypersecretion, and dumping syndrome [32]. Other mechanisms responsible for malabsorption are rapid transit, because of inappropriate mixing of nutrients and reduced mucosal contact for absorption, and small intestinal bacterial overgrowth (SIBO), secondary to alteration in motility and loss of the ileocecal valve. In addition, SIBO worsens malabsorption via direct nutrient consumption (particularly vitamin $\mathrm{B}_{12}$ ) and bile acid deconjugation causing lipid malabsorption [31].

For these reasons, the SBS classification is usually based on anatomical and pathophysiological criteria. The presence of small bowel-colon continuity represents the main factor driving the clinical presentation and determines the differences in the occurrence of possible complications. Colon removal significantly reduces sodium and water absorption increasing the risk of dehydration and renal failure. Furthermore, colon surgery prevents the salvage of calories from unabsorbed carbohydrates, which undergo fermentation by colonic bacteria into short-chain fatty acids (SCFAs) that are absorbed in the colon. On the other hand, the presence of the colon increases the risk of enteric hyperoxaluria, which leads to nephrolithiasis and lactic acidosis [33].

According to the anatomical criteria, 3 types of SBS have been identified:

Type I - end-jejunostomy: patients undergoing end-jejunostomy have the ileum and colon completely removed, while a portion of jejunum is retained and forms the end of the intestine. Patients with a jejunostomy present with accelerated gastric emptying and bowel transit due to reduced hormonal (polypeptide YY, enteroglucagon-1, and enteroglucagon-2) secretion, which in physiological conditions reduces bowel transit. In addition, the increased excretion of water and electrolytes in the lumen is observed to balance endoluminal osmolarity and plasmatic osmolarity. Thus, the patients with end-jejunostomy are at high risk of dehydration immediately after surgery due to large stomal water and sodium losses, which may lead to electrolyte imbalance (particularly hypomagnesemia), hypotension, and renal failure [34].

Type II - jejunocolonic anastomosis: patients with type-II SBS have had a jejuno-ileal resection, in which all or most of the ileum is removed with the preservation of the colon and jejuno-colonic anastomosis formation. Jejunum-colon patients can reabsorb unabsorbed fluids in their colon, thus the clinical picture is dominated by malnutrition, diarrhea/steatorrhea, and vitamin/mineral deficiencies [34].

Type III - jejuno-ileal anastomosis: This type is represented predominantly by jejunal resection leaving $\geq 10 \mathrm{~cm}$ of the terminal ileum and the entire colon intact. Jejunum-ileum patients are uncommon, and they usually do not need nutritional support [34].

As regards the pathophysiological mechanism, SBS can be divided into 2 categories: SBS without the colon and SBS with the colon in continuity.

Finally, in relation to the time interval from SBS development, 3 phases are recognized: acute, adaptation, failure.

The acute phase of SBS lasts 3-4 weeks and involves metabolic derangement and relevant intestinal losses along with increased gastric secretion due to the loss of hormonal negative feedback signals, which are normally released from the terminal ileum. In the acute phase, hospitalization is required for close monitoring to avoid dehydration, acute kidney failure, acid-base imbalance, and electrolyte abnormalities [35]. 
Table 1

Main causes of short bowel syndrome (SBS) in adults.

\begin{tabular}{ll}
\hline Cause of SBS & Relative frequency \\
\hline Surgical complications & $29 \%-50 \%$ \\
Mesenteric infarction (arterial or venous thrombosis) & $25 \%-30 \%$ \\
Neoplasms & $20 \%$ \\
Radiation enteritis & $10 \%$ \\
Crohn's disease & $10 \%-20 \%$ \\
Abdominal trauma & $5 \%-10 \%$ \\
Benign conditions & $5 \%$ \\
Intestinal volvulus & \\
$\quad$ Complicated intussusception & \\
$\quad$ Chronic intestinal pseudo-obstruction & \\
\hline
\end{tabular}

The SBS adaptation phase is a post-resection spontaneous process which lasts $1-2$ years. It is characterized by adaptive changes of the remaining small bowel (structural adaptation) in order to increase the absorptive surface while the time of intestinal transit typically decreases to maximize the time available for absorption (functional adaptation). These processes are promoted by the presence of nutrients in the bowel, pancreatic and biliary secretions, and hormones released by the ileum and colon.

\section{Management of short bowel syndrome}

\subsection{Acute phase}

The SBS acute phase starts immediately after resection and usually lasts for three to four weeks and it is characterized by huge enteric losses. This condition causes a metabolic imbalance with profuse fluid leaks as well as gastric hypersecretion due to the lack of inhibitory hormones normally released from the terminal ileum. Therefore, this condition requires the close monitoring of the patient's total output (both fecal and urinary) and prompt intravenous replacement of fluid and electrolyte losses, even more than protein-energy, in order to prevent any renal impairment, which may eventually occur (Table 1 ). Patients with acute intestinal failure (AIF) are metabolically unstable and need intravenous infusion for days, weeks, or even months and are to be treated in a hospital setting. In this phase both hypo- and hypervolemia ought to be avoided. Initial fluid administration aims to achieve hemodynamic balance, tissue perfusion, and oxygen delivery goals. Severe hypovolemia itself can lead to severe vasoconstriction and activation of the pro-inflammatory cascade. Once hypovolemia has been corrected, vasodilation commonly occurs and should be treated with vasopressors rather than additional fluids. At the same time, treating severe hypovolemia with vasopressors is harmful and the achieved normal blood pressure does not indicate adequate perfusion. Balanced crystalloids should be utilized in the initial resuscitation. Synthetic colloids may expand the intravascular volume more effectively but have been associated with renal dysfunction [36]. The replacement of fluids and electrolytes should be repeated several times a day (up to every $2 \mathrm{~h}$ ) and it seems to better to correct the depletion of liquids and electrolytes with solutions independently from the nutritional support [37]. Whenever possible, the oral administration of rehydrating solutions containing glucose and electrolytes may improve hydrosaline homeostasis.

Parenteral nutrition should promptly begin once the patient stabilizes after surgery. Postoperative ileus usually spontaneously resolves within some days but, while intestinal function recovers, short-term parenteral fluid and nutrition support will be needed. This phase can be self-limiting with the spontaneous recovery of intestinal function, in cases of non-massive bowel resection. Obviously, the duration of parenteral nutrition support depends on the length of the small bowel resected, the location of resection, and the presence of an intact ileocecal valve and colon-in-continuity

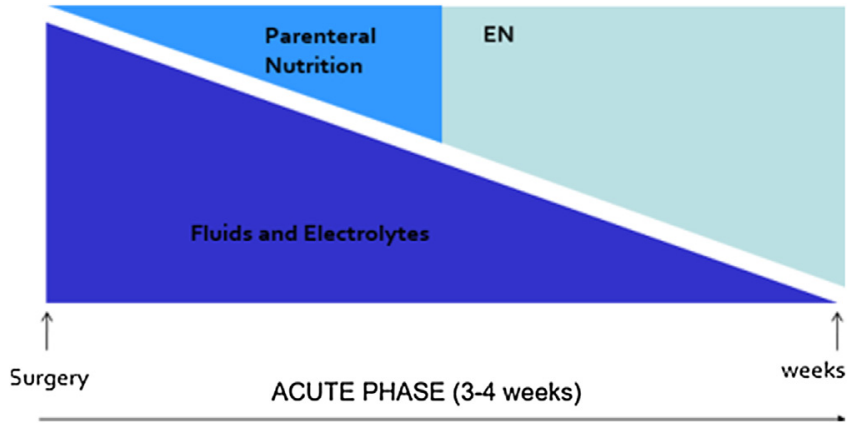

Fig. 1. Schematic management of the acute phase of short bowel syndrome (SBS). $\mathrm{PN}=$ parenteral nutrition.

$\mathrm{EN}=$ enteral nutrition

with the small bowel. This period can be shortened by multimodal enhanced-recovery techniques with the goal to promote the early mobilization and early introduction of oral nutrition and/or enteral nutrition.

Enteral nutrition should even be tried after the stool losses become controllable. In fact, it promotes intestinal adaptation, which in turn improves absorption of nutrients over time. Oral intake is not adequate in most critically ill patients and may carry the risk of aspiration. In the acute phase, any early nutrition aiming to provide the patient's full caloric requirements is harmful, but the optimal amount of calories and protein necessary during this early stage is not well established [37]. Feeding via the enteral route is desirable because it may prevent mucosal atrophy and help to preserve the microbiome, but it is troublesome to monitor malabsorption in this setting. A combined feeding strategy, such as oral-enteral or enteral-parenteral nutrition, should be considered while keeping in mind the possible risk of overfeeding (Fig. 1).

\subsection{Adaptation phase}

The adaptation phase is characterized by structural and functional changes to improve nutrient absorption and slow the gastrointestinal transit. The adaptive phase usually lasts for one to two years. Adaptation is the result of spontaneous adjustments, however, it can be increased through diet, enteral feeding, and medications. The spontaneous post-resection intestinal adaptation is a process that tries to guarantee a more efficient absorption of nutrients per unit length of the remaining gut $[4,34]$. This occurs partly by expanding the absorptive area (structural adaptation) and/or by slowing the gastrointestinal transit (functional adaptation). It is promoted by the presence of nutrients within the gut lumen, by the pancreatic and biliary secretions and by gut hormones, principally produced by the small intestine and colon. Therefore, it is essential throughout this phase that the patients are advised to eat by mouth since this stimulates intestinal absorption and promotes intestinal adaptation [refer to Table 2]. There is no specific diet for individuals with short bowel syndrome. Generally, patients are encouraged to eat at least five or more small meals a day rather than two or three large meals. Concentrated sugars should be avoided or reduced because they can contribute to diarrhea. Some people may need to take additional vitamin or mineral supplementation to interchange for any deficiency of these substances. Likewise, affected individuals might be also given oral rehydration solutions to keep the correct fluid balance up. These solutions, composed of water, sugar, and salt, in some cases, can reduce the necessity for parenteral nutrition. The affected individuals may also be treated by means of enteral nutrition. Thus, not all the patients with SBS are intended to intestinal failure, as this condition may be reversible. Post-operative intestinal adaptation appears to be 
Table 2

Main characteristic of the different type of SBS according to the anatomical criteria.

\begin{tabular}{|c|c|c|c|}
\hline & Type I (end-jejunostomy) & $\begin{array}{l}\text { Type II (jejuno-colonic } \\
\text { anastomosis) }\end{array}$ & $\begin{array}{l}\text { Type III (jejuno-ileal } \\
\text { anastomosis) }\end{array}$ \\
\hline Surgical procedure & $\begin{array}{l}\text { Complete resection of } \\
\text { ileum and colon. Jejunum } \\
\text { preserved and represent } \\
\text { the end of the intestine. }\end{array}$ & $\begin{array}{l}\text { Resection of most of the } \\
\text { ileum with preservation of } \\
\text { colon. }\end{array}$ & $\begin{array}{l}\text { Jejunal resection, at least } \\
10 \mathrm{~cm} \text { of terminal ileum } \\
\text { and colon are retained. }\end{array}$ \\
\hline Representation & & & thant \\
\hline $\begin{array}{l}\text { Presence of ileum-coecal } \\
\text { valve }\end{array}$ & No & No & Yes \\
\hline Preservation of colon & No & Partial & Complete \\
\hline Clinical features & $\begin{array}{l}\text { Dehydration immediately } \\
\text { postsurgery with risk of } \\
\text { electrolyte imbalances. } \\
\text { Jejunal output increase } \\
\text { after food and drink intake }\end{array}$ & $\begin{array}{l}\text { Weight loss, } \\
\text { diarrhea/steatorrhea. } \\
\text { Severe malnutrition } \\
\text { developing in months. }\end{array}$ & $\begin{array}{l}\text { Malnutrition rare. Usually } \\
\text { not need for parenteral } \\
\text { support }\end{array}$ \\
\hline
\end{tabular}

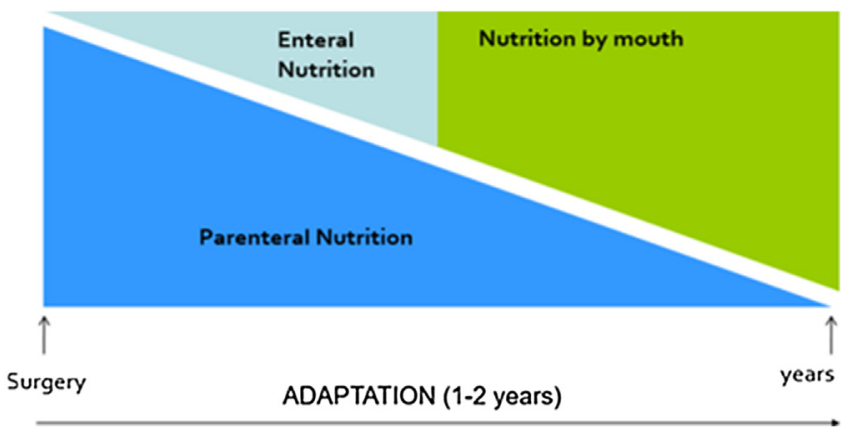

Fig. 2. Schematic management of the adaptation phase of short bowel syndrome (SBS).

$\mathrm{PN}=$ parenteral nutrition.

$\mathrm{EN}=$ enteral nutrition.

absent or impaired in the presence of an end-jejunostomy [4,34] (Fig. 2).

\subsection{Chronic intestinal failure}

SBS-associated intestinal failure reverses completely in approximately $50 \%$ of adults within the first two years. Thereafter, important intestinal adaptation occurs in only a minority of patients. In the absence of further intervention (e.g. trophic factors, autologous gastrointestinal reconstruction, and intestinal transplantation) these patients remain dependent on chronic parenteral nutrition.

Around fifty percent of prolonged acute intestinal failure evolves to chronic intestinal failure (CIF) [38]. Patients with CIF are metabolically stable, need intravenous nutritional support over months or years (reversible CIF) or lifelong (irreversible CIF) and are treated by home-based parenteral nutrition (HPN). During this phase symptoms of short bowel syndrome are usually addressed with medication (Table 3).

Overall, the probability of weaning off HPN has been reported to be about $50 \%$ in adults and up to $73 \%$ in children. Complete com-
Table 3

Medications used in the late phase of short bowel syndrome.

Anti-diarrheal medication (e.g. loperamide, codeine)

Pancreatic enzyme replacement therapy

Bile-acid resins, such as cholestyramine

$\mathrm{H} 2$ blocker and proton-pump inhibitors to reduce stomach acid

Antibiotics for bacterial overgrowth

Lactase supplement (to improve the bloating and diarrhea associated with lactose intolerance)

Drugs that reduce the frequency and volume of total parenteral nutrition (TPN), comprising:

oral solution of glutamine

growth hormone, of recombinant DNA origin, for injection together with a specialized oral diet.

teduglutide, a glucagon-like peptide-2 analog

mutation off HPN in patients with SBS is unlikely [38]. Parenteral nutrition can be necessary either to replace the total caloricnutritional needs when patients are on fasting and are totally dependent on HPN (total PN) or to replace only a part of it when patients partially satisfy the energy requirement by oral/enteral feeding (supplemental PN).

\section{Short bowel syndrome complications and consequences}

Short bowel syndrome complications can occur as a consequence of SBS itself as well as complications of long-term HPN.

\subsection{Consequences related to short bowel syndrome (SBS)}

\subsubsection{Diarrhea}

The physiological modifications in SBS lead to numerous potential clinical alterations. Diarrhea tends to be the most debilitating symptom for the majority of patients with SBS. Uncontrolled and refractory diarrhea is the symptom of the greatest negative impact on the quality of life of HPN patients [39]. Therefore, the effective management of diarrhea is of primary importance to this population of patients. Many pathophysiological mechanisms are at the base of diarrhea, such as gastric hypersecretion, which increases 
the fluid amount, and acidity and denatures and inactivates pancreatic enzymes, and bile salts, which contribute to maldigestion and malabsorption. Moreover, a diminished bile salt pool resulting from distal ileum resection further aggravates malabsorption. Again, because of to the intestinal resection reduced gut hormone feedback mechanisms (e.g. peptide YY, glucagon-like peptide-1) facilitate accelerated dumping into the upper gut with rapid intestinal transit. Finally, small bowel bacterial overgrowth, as well as the primary disease that caused SBS (Chron's disease, radiation enteritis), may contribute to aggravate diarrhea.

\subsubsection{Intestinal failure-associated liver disease (IFALD)}

A liver injury, named intestinal failure-associated liver disease (IFALD), is a potential complication for patients on long-term HPN. Alterations of liver function occurring in patients on PN were initially defined as PN-associated liver disease (PNALD) [40]. However, increasing evidence has suggested that liver disease is not linked to HPN per se, but rather to underlying intestinal failure (IF), hence the term IFALD. Accordingly, the European Society for Clinical Nutrition and Metabolism (ESPEN) position paper on IFALD recommends that "the term IFALD refers to liver injury as a result of one or more factors relating to IF, including, but not limited to, PN and occurring in the absence of another primary parenchymal liver pathology (e.g. viral or autoimmune hepatitis), other hepatotoxic factors (e.g. alcohol/medication) or biliary obstruction" [41].

The IFALD pathogenesis is multifactorial and mainly related to several factors: (a) increased intestinal permeability due to the mucosal atrophy related to the lack of enteral/oral feeding; (b) alteration of the entero-hepatic circulation of bile acids; and (c) changes in the gut microbiome, causing the latter conditions the disruption of the gut-liver axis [38].

Infants seem to be more at risk of IFALD than adults and accordingly, cholestasis is more frequent in infants, in whom the evolution to severe hepatic fibrosis, cirrhosis, and end-stage liver failure is more rapid.

No formally agreed criteria have been defined so far to diagnose IFALD, even if a persistent elevation of liver function tests (LFTs), alkaline phosphatase and $\gamma$-glutamyl transferase $\geq 1.5$ above the upper limit, normal or conjugated bilirubin $>0.3 \mathrm{mg} / \mathrm{dL}$, which persists for $\geq 6$ months in adults or $\geq 6$ weeks in children, are widely accepted criteria $[42,43]$. Liver histology is not considered mandatory for the diagnosis of IFALD and the need for it should be evaluated on a case-by-case basis.

Depending on the used diagnostic criteria, a wide range of prevalence and incidence ( $0 \%-50 \%$ ) of IFALD have been reported, in particular the range of the frequency of IFALD was $5 \%-15 \%$ for cholestasis, $19 \%-43 \%$ for steatosis, $11 \%-22 \%$ for fibrosis, and $7 \%-40 \%$ for unclassified criteria [44].

IFALD can ultimately progress to advanced liver disease up to end-stage liver failure, which can represent a mandatory indication for life-saving combined liver and small bowel transplantation. Monitoring the evolution of IFALD is therefore essential to prevent the evolution to end-stage liver disease as well as for planning a patient's referral for liver transplantation. No association was found between the liver function tests (LFTs) and the histological degree of fibrosis. The role of transient elastography (TE) as a non-invasive marker of liver fibrosis in various liver diseases for patients on HPN has been evaluated in only a few studies, with conflicting results: in fact TE values seems to be significantly correlated to the serum bilirubin level and severity of histological cholestasis, more than irreversible fibrosis in adult patients [45], whereas according to a study on children, TE has shown that it can be a promising non-invasive method for monitoring the development of IF-related liver histopathology [46]. However, according to TE results, the prevalence of advanced fibrosis or cirrhosis during HPN, was low $(<10 \%)$ without further significant progression
Table 4

ESPEN guidelines: recommendations for the prevention of IFALD

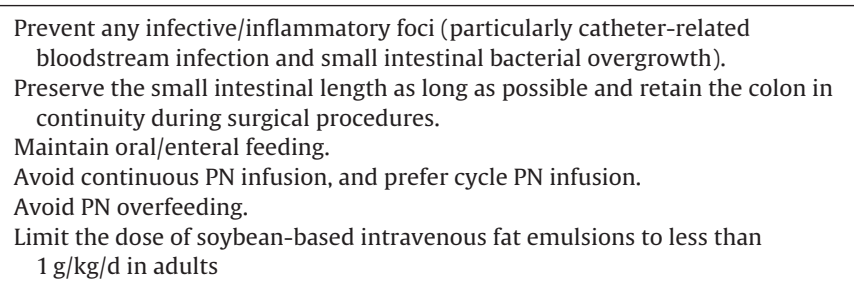

within the 12-month follow-up and the short intestinal remnant length $<100 \mathrm{~cm}$ appeared to be a risk factor for the development of fibrosis [47]

The recommendations from the ESPEN guidelines about the prevention of IFALD are summarized in Table 4.

\subsubsection{Other metabolic complications of SBS}

Patients with SBS may experience a number of other complications, such as cholelithiasis, oxalate nephropathy, and D-lactic acidosis. Cholelithiasis, particularly cholesterol stones, can occur in up to $44 \%$ of patients with SBS $[10,48]$. Risk factors for cholelithiasis include the use of parenteral nutrition, resection of the terminal ileum, and an intestinal remnant $<120 \mathrm{~cm}[10,48,49]$.

The pathogenesis of cholelithiasis in patients with SBS is not completely understood. At least two factors may contribute: altered bile composition, due to the entero-hepatic circulation of bile acids impaired by ileal resection, results in a reduction in hepatic bile acid secretion which becomes supersaturated with cholesterol; another possible mechanism can be the bile stasis, due to the diminished enteric hormonal stimulation of gallbladder contractions that may lead to biliary stasis and biliary sludge formation.

It has been reported that the risk of complications of cholelithiasis may be higher in patients with SBS, as compared with the general population, therefore prophylactic cholecystectomy may be taken into consideration [50].

Patients with SBS and a colon in continuity with intestinal contents are at increased risk for oxaluria and nephrolithiasis. In SBS patients with fat malabsorption and a colon in continuity, calcium binds to unabsorbed fatty acids, leaving oxalate free to pass into the colon to be absorbed and then filtered by the kidney. In the kidney, oxalate binds to calcium, resulting in oxalate nephrolithiasis and progressive obstructive nephropathy. The management of patients with enteric hyperoxaluria is through a low-oxalate diet, high fluid intake, potassium citrate to correct metabolic acidosis if present, and oral calcium carbonate to bind oxalate in the intestinal lumen.

Another possible complication is related to D-lactic acidosis, a rare neurological syndrome characterized by altered mental status ranging from confusion to coma, slurred speech, seizures, and ataxia resulting from bacterial fermentation of unabsorbed carbohydrates. It primarily occurs in patients with an intact colon and often in the setting of underlying bacterial overgrowth. In such patients, the delivery of unabsorbed carbohydrates to the colon causes gram-positive anaerobic bacteria to produce D-lactate (rather than L-lactate that is produced by normal metabolic processes).

\subsection{Consequences related to long-term HPN}

The long-term use of TPN can be associated with a variety of complications including bacterial infections, intravenous catheter complications, low bone calcium uptake, blood clots, gallbladder disease, kidney disease, and liver problems. Liver and kidney problems can ultimately result in liver or kidney failure. 


\subsubsection{Central venous access device (CVAD)-related complications}

Central venous access device infection is a significant complication in patients receiving long-term HPN [51,52]. The most common complications for HPN therapy are CVAD mechanical complications and catheter-related bloodstream infection (CRBSI).

The CVADs used for HPN infusion include total implanted infusion venous access devices (TCVC), PICCs, and tunneled catheters. These types of catheters carry different rates of complications. As concerns mechanical complications, it seems that the risk for these complications does not differ by the type of CVAD. It seems that PICCs are associated with an increased risk for deep vein thrombosis, limiting their use for indefinite PN therapy and situations where vessel preservation is a priority. According to recent guidelines, and based on observational studies and expert consensus, tunneled CVADs should be selected for adult patients requiring long-term daily PN infusions. If the duration of HPN is uncertain or of short duration (i.e. < one month), PICCs may be used, even if based on a low grade of evidence [53].

As concerns infective complications, catheter-related bloodstream infection (CRBSI) is an important and still too common complication of parenteral nutrition. A recent meta-analysis has showed a lower rate of catheter-related bloodstream infection in HPN patients using PICC compared with TCVC, but only when analyzing comparative studies. However, the analysis of singlearm studies has shown that the rate of CRBSI was comparable in PICC and TCVC use [54]. Predictive factors significantly related to catheter-related bloodstream infection in patients receiving homebased parenteral nutrition are longer duration of HPN, cyclic HPN (compared with continuous HPN), and hospital-based compound HPN (compared with 3-in-1 commercial formulations), all being significantly associated with CRBSI [52].

The risk of infection can be reduced by adopting cost-effective, evidence-based interventions such as proper education and specific training of the ward personnel, an adequate hand washing policy, proper choice-making regarding the type of device and site of insertion, use of maximal barrier protection during insertion, use of chlorhexidine as an antiseptic prior to insertion and for disinfecting the exit site thereafter, appropriate policies for dressing the exit site, routine changes of administration sets, and removal of central lines as soon as they are no longer necessary.

Long-term catheters should be removed from patients with CRBSI only when associated with such complications as severe sepsis, suppurative thrombophlebitis, endocarditis, bloodstream infection that continues in spite of 72 -h antimicrobial therapy to which the infecting microbes are susceptible. In uncomplicated CRBSI involving long-term catheters due to pathogens other than $S$. aureus, $P$. aeruginosa, Bacillus species, Micrococcus species, Propionibacteria, fungi, or mycobacteria, because of the limited access sites in many patients with SBS, treatment should be attempted without catheter removal and with application of both systemic and antimicrobial lock therapy [55]. In fact, the expansive duration of HPN (ranging from months to decades) has shifted the focus of care to salvaging rather than removing the CVAD when infected. Infusion of concentrated antibiotics (antibiotic lock therapy) sensitive to the offending organisms into the CVAD lumen should be used for catheter salvage. Urokinase and other thrombolytic agents are not recommended as adjunctive therapy for patients with CRBSI [55].

Prophylactic antibiotic lock solutions are only applicable to patients with long-term CVADs who have a history of multiple CRBSI despite optimal maximum adherence to aseptic technique. Ethanol and antimicrobial lock instillations should be considered when needed to prevent recurrent infection [53]. Tunneled CVADs instilled with concentrated vancomycin have demonstrated a decrease in CRBSI in one study [56]. Recent studies have also shown that taurolidine lock is a cost-effective intervention in patients on HPN with a high risk of CRBSI [57-59].

In general, the strict compliance by patients and caregivers with evidence-based instructions together with supervision by welltrained HPN providers is the most effective strategy to prevent CVAD-related complications. The type of CVAD should be selected based upon the length of therapy, patient choice, and the ability of the patient/caregiver to care for the CVAD, as well a patient's underlying comorbidities that may potentially contribute to other catheter-related complications.

\subsection{Other metabolic complications of HPN}

Other metabolic complications described during long-term HPN can be represented by metabolic bone disease, iron deficiency anemia, manganese toxicity. There are a number of treatment options for each of these complications. It is important to evaluate the entire clinical picture prior to initiating an intervention and consider evidence-based interventions when available.

\subsubsection{Metabolic bone disease (MBD)}

Low bone mineral density with metabolic bone disease (MBD) is commonly reported in patients receiving home-based parenteral nutrition. Osteoporosis contributes to morbidity in patients with intestinal failure on long-term HPN. The pathogenesis of MBD is related to several factors which may be HPN-related and/or related to a patient's underlying disease. Patients affected by SBS obviously present with poor nutrient absorption, particularly fat malabsorption, which may play a role in vitamin D deficiency [60]. Moreover, inadequate ultraviolet exposure from sunlight due to chronic illness and medications that affect the vitamin D metabolism are additional risk factors found in HPN patients [61]. The prevalence of osteoporosis in HPN patients with IF has been found to range from 57\% [62] to 67\% [63]. Low BMI and vitamin-D deficiency were identified as independent risk factors.

Giving the high prevalence of MBD, it is therefore important to monitor the vitamin D levels during HPN [64] at least every six months, and adequately supplement it by intramuscular or separate vitamin $\mathrm{D}$ parenteral infusion, even if the exact dose and duration of oral vitamin D supplementation in intestinal failure patients has not been completely established. Moreover, in patients on long-term HPN, BMD should be annually monitored and osteoporosis should be treated [64]. From a therapeutic point of view, oral and intravenous calcium, vitamin $\mathrm{D}$, and bisphosphonates have been used to treat BMD even if with low efficiency due to their limited absorption and patients' compliance. Also, vitamin K supplementation should be taken into consideration as it may have a potential benefit to BMD. More recently, denosumab, a new drug that helps to prevent osteoclast development and activation and led to decreased bone resorption, has been studied in patients with intestinal failure receiving HPN with encouraging results [65].

\subsubsection{Iron deficiency anemia}

Patients receiving long-term HPN have been reported with a high incidence of iron deficiency anemia, which varies from $30 \%$ to $55 \%$ of cases $[66,67]$.

Nevertheless, iron has not been routinely added to HPN with the exception of a small entity of iron being a component of the main supplements of trace elements. Instead, intravenous iron preparations are not currently used, because of the reported risks of anaphylaxis and because the data regarding the compatibility of iron supplementation with parenteral mixtures are conflicting [67].

Iron status should be assessed before HPN initiation and routinely monitored during therapy. 


\subsubsection{Manganese toxicity}

Manganese (Mn) is an essential micronutrient required for normal physiological processes, such as bone growth, development of cartilage and connective tissues, reproductive function, neuronal function [68]. It is a fundamental component of parenteral nutrition, however, its requirement is low and the iatrogenic risks associated with excessive Mn administration in HPN patients are well documented [68]. The ideal Mn equilibrium is hard to obtain and it is even more difficult to assess it with the commonest method of measurement of its concentration in the whole blood [69]. The data in the literature is conflicting about the best method for assessing Mn stores in humans and a definitive biomarker of Mn exposure or induced neurotoxicity is yet to be identified or validated.

Despite all the described essential physiological functions of Mn and its classification as an essential trace element, there is little evidence in humans of Mn deficiency being clinically relevant [70], as only a few cases of Mn deficiency have been described in humans receiving unsupplemented $\mathrm{PN}$. Instead, more relevant is this risk of excessive Mn administration. Mn toxicity usually presents with parkinsonian-like symptoms and this risk can even increase in case of cholestasis or anemia or, of course, if the patient has received high doses of Mn [68]. Hypermanganesemia and neurotoxicity are in fact associated with the duration of Mn supplementation and Mn dosage [68]. Most of the case reports on Mn intoxication were about adults receiving $>500 \mu \mathrm{g} / \mathrm{d}$ parenteral Mn. The currently recommended dose of Mn for adults treated with PN is $55 \mu \mathrm{g} / \mathrm{d}$, but the doses provided by most currently available multi-trace element products exceed this.

\section{Short bowel therapeutic approaches}

\subsection{Drugs that reduce the frequency and volume of total parenteral nutrition}

\subsubsection{Oral solution of glutamine}

Glutamine is an amino acid that is well and actively absorbed in the human jejunum. Historical studies have demonstrated that glutamine, like and together with glucose, stimulates both neutral and electrogenic sodium absorption. Classically, in patients with cholera, glutamine together with glucose significantly reduces net water and sodium secretion [71]. Again, oral rehydration solutions with glutamine and glucose are widely used to treat the dehydration associated with diarrhea in infants. In addition, glutamine contributes to intestinal cell proliferation by augmenting the effects of growth factors such as epidermal growth factor (EGF), insulin-like growth factor-I (IGF-I) and transforming growth factor$\alpha$ (TGF- $\alpha$ ) [72]. For these reasons, glutamine has been used and studied in patients with short-bowel syndrome, however despite the pathophysiological assumptions, there is no current evidence that glutamine can be used effectively to reduce the amount of gastrointestinal fluid losses. To date, studies performed have failed to demonstrate any beneficial effect of glutamine [73].

\subsubsection{Growth hormone}

Somatropin (of recombinant DNA origin) for injection, a human growth hormone, has been approved by the Food and Drug Administration (FDA) for the treatment of patients with SBS. Under the influence of trophic factors, such as growth hormone $(\mathrm{GH})$, intestinal adaptation is enhanced. Somatropin is similar to the $\mathrm{GH}$ produced by the pituitary gland. This drug can improve the ability of the small intestine to absorb nutrients, thereby reducing the need for TPN. Supplying a therapeutic regimen of GH, an optimized diet and glutamine supplementation for 4 weeks to patients with short bowel syndrome who are dependent on parenteral nutrition, has shown to reduce their long-term requirements for parenteral nutrition $[74,75]$.

\subsubsection{Glucagon-like peptide-2 analog}

Teduglutide was approved by the U.S. Food and Drug Administration (FDA) in 2012 to treat patients with short bowel syndrome, aged $\geq 1$ year, who are stable following a period of post-surgery intestinal adaptation. This drug is a glucagon-like peptide-2 analog that increases intestinal absorption.

In a phase-III trial on adults with SBS IF dependent on homebased parenteral nutrition, a significantly greater proportion of patients taking $0.05 \mathrm{mg} / \mathrm{kg} / \mathrm{day}$ teduglutide than placebo recipients achieved $\mathrm{a} \geq 20 \%$ reduction in weekly HPN volume from baseline to week 20 and maintained it to week 24 [76]. These results were subsequently confirmed in a real-life setting as well [77], and several studies have demonstrated that teduglutide can be applied to anatomically and clinically heterogeneous SBS-IF patients and it results in an adaptive response with variable time and effect range in routine medical care [78]. In real-life experience, in contrast to randomized controlled studies, the reduction of parenteral support has taken longer [77].In this study stool consistency and frequency, as well as the sensation of thirst, were identified as early clinical markers of response [77]. Additionally, a recent study has demonstrated that HPN volume reductions were associated with improvements in quality of life scores for patients with short bowel syndrome treated with teduglutide [79]. Adverse events were mostly of mild-to-moderate severity and generally consistent with the underlying condition or known mechanism of the drug (e.g. central line-related issues, gastrointestinal events). Teduglutide is, therefore, a useful treatment option.

\subsection{Surgical procedures}

Surgical treatments other than transplantation have been proposed in order to increase the absorptive area, slowing down the intestinal transit and reducing bacterial overgrowth.

Surgical procedures to lengthen the small bowel include the Bianchi procedure, which consists in a longitudinal incision of a dilated segment of small bowel to then sew the two new sections end to end; the serial transverse enteroplasty (STEP), where the bowel is incised and stapled in a diagonal non-occlusive pattern in order to increase the bowel length and slow transit time; and the spiral intestinal lengthening and tailoring (SILT), a spiral incision around the dilated small bowel then re-sewn to tailor the length [80]. Gastrointestinal reconstructive procedures have been described mostly in pediatric SBS. One of the largest adult series of 20 patients over a 15 -year period, has shown a mean length of gut increased from $60 \mathrm{~cm}$ to $80 \mathrm{~cm}$ post-surgery with weaned-off $\mathrm{PN}$ or increased enteral calorie intake for 17 patients [81]. All the reconstructive techniques harbor a relevant risk of anastomotic breakdown, stricture, and vascular injury. For these reasons, SBS surgical approaches are usually performed in highly specialized centers [82].To date, reconstructive procedures have been performed mostly on children and their application in adults should be reserved to specifically selected cases.

\subsection{Intestinal transplantation}

Over the last years, intestinal transplantation has become a viable option for patients with SBS and intestinal failure. The terminal ileum represents the used graft, because of technical feasibility and its greater adaptive potential than the jejunum [83]. To date, intestinal transplant may be considered for highly selected patients who are likely to experience a poor outcome on long-term PN (ultra-short residual small intestinal length $<50 \mathrm{~cm}$, end-jejunostomy, and patients with motility disorders) $[17,34,84]$. 
It does not represent an alternative therapy for stable patients on intravenous nutrition, because of both the excellent outcomes reported for long-term parenteral nutrition and the challenges of intestinal transplantation [85]. Indeed, intestine transplant often requires a liver-to-small-bowel graft or a "multivisceral" graft (such as the stomach and pancreas) with liver-containing grafts demonstrated having the longest survival [86]. In addition, the small bowel is an arduous organ to transplant due to its immunogenicity, large population of donor immune cells present within the graft, and its non-sterile contents which increases both the risk of rejection and infection [34]. It is mostly thanks to the recent advances in immunosuppression that short-term results of small bowel transplant have recently improved in terms of survival and digestive autonomy [87]. The challenges for long-term results are chronic rejection and immunosuppressant-related complications [88]. Currently, the one-year survival rate for intestinal transplantation approaches $80 \%$, while the 5 -year survival rate is approximately $50 \%$, with the majority of survivors being free of parenteral nutrition [34]. In conclusion, intestinal transplantation should be suggested to a very select subset of SBS patients with severe and irreversible complications of PN and no hope of intestinal rehabilitation.

\section{Conclusions}

SBS is a complex and articulated condition, with many shades and clinical implications. Its management and treatment usually involve gastroenterologists and nutrition specialists working together as a multi-disciplinary team.

\section{Conflict of interest}

None declared.

\section{Appendix A. Supplementary data}

Supplementary material related to this article can be found, in the online version, at doi:https://doi.org/10.1016/j.dld.2019.11. 013.

\section{References}

[1] Carlsson E, Bosaeus I, Nordgren S. Quality of life and concerns in patients with short bowel syndrome. Clin Nutr 2003;22:445-52.

[2] Pironi L, Arends J, Baxter J, Bozzetti F, Peláez RB, Cuerda C, et al. ESPEN endorsed recommendations. Definition and classification of intestinal failure in adults. Clin Nutr 2015;34:171-80.

[3] DiBaise J. Home parenteral nutrition: complications, survival, costs, and quality of life. In: Langnas A, Goulet O, Quigley E, Tappenden K, editors. Intestinal failure: diagnosis, management, and transplantation. Oxford: Blackwell Publishing; 2008. p. 130-41.

[4] Buchman AL, Scolapio J, Fryer J. AGA technical review on short bowel syndrome and intestinal transplantation. Gastroenterology 2003;124:1111-34

[5] O'Keefe S], Buchman AL, Fishbein TM, Jeejeebhoy KN, Jeppesen PB, Shaffer J. Short bowel syndrome and intestinal failure: consensus definitions and overview. Clin Gastroenterol Hepatol 2006;4:6-10.

[6] DiBaise JK, Young RJ, Vanderhoof JA. Intestinal rehabilitation and the short bowel syndrome: part 1. Am J Gastroenterol 2004;99:1386-95.

[7] Jeppesen PB. Teduglutide, a novel glucagon-like peptide 2 analog, in the treatment of patients with short bowel syndrome. Therap Adv Gastroenterol 2012:5:159-71.

[8] Brandt CF, Hvistendahl M, Naimi RM, Tribler S, Staun M, Brøbech P, et al. Home parenteral nutrition in adult patients with chronic intestinal failure: the evolution over 4 decades in a tertiary referral center. JPEN J Parenter Enteral Nutr 2017;41:1178-87.

[9] Thompson JS. Comparison of massive vs. repeated resection leading to short bowel syndrome. J Gastrointest Surg 2000;4:101-4.

[10] Nightingale JM, Lennard-Jones JE, Gertner DJ, Wood SR, Bartram CI. Colonic preservation reduces need for parenteral therapy, increases incidence of renal stones, but does not change high prevalence of gall stones in patients with a short bowel. Gut 1992;33:1493-7.

[11] Amiot A, Messing B, Corcos O, Panis Y, Joly F. Determinants of home parenteral nutrition dependence and survival of 268 patients with non-malignant short bowel syndrome. Clin Nutr 2013;32:368-74.
[12] Thompson JS. Short bowel syndrome and malabsorption - causes and prevention. Viszeralmedizin 2014;30:174-8.

[13] Grainger JT, Maeda Y, Donnelly SC, Vaizey CJ. Assessment and management of patients with intestinal failure: a multidisciplinary approach. Clin Exp Gastroenterol 2018;11:233-41.

[14] Carlson GL, Dark P. Acute intestinal failure. Curr Opin Crit Care 2010;16:347-52

[15] Thompson JS, DiBaise JK, Iyer KR, Yeats M, Sudan DL. Postoperative short bowel syndrome. J Am Coll Surg 2005;201:85-9.

[16] McBride CL, Oleynikov D, Sudan D, Thompson JS. Short bowel syndrome after laparoscopic procedures. Am Surg 2014;80:382-5.

[17] Messing B, Crenn P, Beau P, Boutron-Ruault MC, Rambaud JC, Matuchansky C. Long-term survival and parenteral nutrition dependence in adult patients with the short bowel syndrome. Gastroenterology 1999;117:1043-50.

[18] Vantini I, Benini L, Bonfante F, Talamini G, Sembenini C, Chiarioni G, et al. Survival rate and prognostic factors in patients with intestinal failure. Dig Liver Dis 2004;36:46-55.

[19] Gupta PK, Natarajan B, Gupta H, Fang X, Fitzgibbons RJ. Morbidity and mortality after bowel resection for acute mesenteric ischemia. Surgery 2011;150:779-87.

[20] Alhan E, Usta A, Çekiç A, Saglam K, Türkyılmaz S, Cinel A. A study on 107 patients with acute mesenteric ischemia over 30 years. Int J Surg 2012;10:510-3.

[21] Sanders BM, Dalsing MC. Mesenteric ischemia affects young adults with predisposition. Ann Vasc Surg 2003;17:270-6.

[22] Sudhakar CB, Al-Hakeem M, MacArthur JD, Sumpio BE. Mesenteric ischemia secondary to cocaine abuse: case reports and literature review. Am J Gastroenterol 1997;92:1053-4.

[23] Thompson JS, Weseman R, Rochling F, Grant W, Botha J, Langnas A, et al. Radiation therapy increases the risk of hepatobiliary complications in short bowel syndrome. Nutr Clin Pract 2011;26:474-8.

[24] Boland E, Thompson J, Rochling F, Sudan D. A 25-year experience with postresection short-bowel syndrome secondary to radiation therapy. Am J Surg 2010;200:690-3.

[25] Wheeler M, Mercer D, Grant W, Botha J, Langnas A, Thompson J. Surgical treatment of intra-abdominal desmoid tumors resulting in short bowel syndrome. Cancers (Basel) 2012;4:31-8.

[26] Thompson JS, Iyer KR, DiBaise JK, Young RL, Brown CR, Langnas AN. Short bowel syndrome and Crohn's disease. J Gastrointest Surg 2003;7:1069-72.

[27] Elriz K, Palascak-Juif V, Joly F, Seguy D, Beau P, Chambrier C, et al. Crohn's disease patients with chronic intestinal failure receiving long-term parenteral nutrition: a cross-national adult study. Aliment Pharmacol Ther 2011;34:931-40.

[28] Uchino M, Ikeuchi H, Bando T, Matsuoka H, Takahashi Y, Takesue Y, et al. Risk factors for short bowel syndrome in patients with Crohn's disease. Surg Today 2012;42:447-52.

[29] Thompson JS, Gilroy R, Sudan D. Short bowel syndrome after continencepreserving procedures. J Gastrointest Surg 2008;12:73-6.

[30] Dabney A, Thompson J, DiBaise J, Sudan D, McBride C. Short bowel syndrome after trauma. Am J Surg 2004;188:792-5.

[31] Bielawska B, Allard JP. Parenteral nutrition and intestinal failure. Nutrients 2017;9:E466.

[32] Nightingale JM, Kamm MA, van der Sijp JR, Ghatei MA, Bloom SR, Lennard-Jones JE. Gastrointestinal hormones in short bowel syndrome. Peptide YY may be the' colonic brake' to gastric emptying. Gut 1996;39:267-72.

[33] Jeppesen PB, Mortensen PB. Significance of a preserved colon for parenteral energy requirements in patients receiving home parenteral nutrition. Scand J Gastroenterol 1998;33:1175-9.

[34] Nightingale J, Woodward JM, Gastroenterology Small Bowel and Nutrition Committee of the British Society of Gastroenterology. Guidelines for management of patients with a short bowel. Gut 2006;55:1-12.

[35] Pironi L. Definitions of intestinal failure and the short bowel syndrome. Best Pract Res Clin Gastroenterol 2016;30:173-85.

[36] Kashy BK, Podolyak A, Makarova N, Dalton JE, Sessler DI, Kurz A. Effect of hydroxyethyl starch on postoperative kidney function in patients having noncardiac surgery. Anesthesiology 2014;121:730-9.

[37] Pironi L, Corcos O, Forbes A, Holst M, Joly F, Jonkers C, et al. Intestinal failure in adults: recommendations from the ESPEN expert groups. Clin Nutr 2018;37:1798-809.

[38] Pironi L, Sasdelli AS. Intestinal failure-associated liver disease. Clin Liver Dis 2019:23:279-91.

[39] Winkler MF, Hagan E, Wetle T, Smith C, Maillet JO, Touger-Decker R. An exploration of quality of life and the experience of living with home parenteral nutrition. JPEN J Parenter Enteral Nutr 2010;34:395-407.

[40] Buchman AL, Iyer K, Fryer J. Parenteral nutrition-associated liver disease and the role for isolated intestine and intestine/liver transplantation. Hepatology 2006;43:9-19.

[41] Lal S, Pironi L, Wanten G, Arends J, Bozzetti F, Cuerda C, et al. Clinical approach to the management of Intestinal Failure Associated Liver Disease (IFALD) in adults: a position paper from the Home Artificial Nutrition and Chronic Intestinal Failure Special Interest Group of ESPEN. Clin Nutr 2018;37:1794-7.

[42] Cavicchi M, Beau P, Crenn P, Degott C, Messing B. Prevalence of liver disease and contributing factors in patients receiving home parenteral nutrition for permanent intestinal failure. Ann Intern Med 2000;132:525-32.

[43] Pironi L, Konrad D, Brandt C, Joly F, Wanten G, Agostini F, et al. Clinical classification of adult patients with chronic intestinal failure due to benign disease: an international multicenter cross-sectional survey. Clin Nutr 2018;37:728-38.

[44] Sasdelli AS, Agostini F, Pazzeschi C, Guidetti M, Lal S, Pironi L. Assessment of intestinal failure associated liver disease according to different diagnostic criteria. Clin Nutr 2019;38:1198-205. 
[45] Van Gossum A, Pironi L, Messing B, Moreno C, Colecchia A, D'Errico A, et al. Transient elastography (FibroScan) is not correlated with liver fibrosis but with cholestasis in patients with long-term home parenteral nutrition. JPEN J Parenter Enteral Nutr 2015:39:719-24.

[46] Hukkinen M, Kivisaari R, Lohi J, Heikkilä P, Mutanen A, Merras-Salmio L, et al. Transient elastography and aspartate aminotransferase to platelet ratio predict liver injury in paediatric intestinal failure. Liver Int 2016;36:361-9.

[47] Knop V, Neuberger SC, Marienfeld S, Bojunga J, Herrmann E, Poynard T, et al. Intestinal failure-associated liver disease in patients with short bowel syndrome: evaluation by transient elastography. Nutrition 2019;63-64:134-40.

[48] Roslyn JJ, Pitt HA, Mann LL, Ament ME, DenBesten L. Gallbladder disease in patients on long-term parenteral nutrition. Gastroenterology 1983;84:148-54.

[49] Dray X, Joly F, Reijasse D, Attar A, Alves A, Panis Y, et al. Incidence, risk factors, and complications of cholelithiasis in patients with home parenteral nutrition. J Am Coll Surg 2007;204:13-21.

[50] Thompson JS. The role of prophylactic cholecystectomy in the short-bowel syndrome. Arch Surg 1996;131:556-9.

[51] Wouters Y, Roosenboom B, Causevic E, Kievit W, Groenewoud H, Wanten GJA. Clinical outcomes of home parenteral nutrition patients using taurolidine as catheter lock: a long-term cohort study. Clin Nutr 2019;38:2210-8.

[52] Pichitchaipitak O, Ckumdee S, Apivanich S, Chotiprasitsakul D, Shantavasinkul PC. Predictive factors of catheter-related bloodstream infection in patients receiving home parenteral nutrition. Nutrition 2018;46:1-6.

[53] Kovacevich DS, Corrigan M, Ross VM, McKeever L, Hall AM, Braunschweig C. American society for parenteral and enteral nutrition guidelines for the selection and care of central venous access devices for adult home parenteral nutrition administration. JPEN J Parenter Enteral Nutr 2019;43:15-31.

[54] Hon K, Bihari S, Holt A, Bersten A, Kulkarni H. Rate of catheter-related bloodstream infections between tunneled central venous catheters versus peripherally inserted central catheters in adult home parenteral nutrition: a meta-analysis. JPEN J Parenter Enteral Nutr 2019;43:41-53.

[55] Mermel LA, Allon M, Bouza E, Craven DE, Flynn P, O'Grady NP, et al. Clinical practice guidelines for the diagnosis and management of intravascular catheter-related infection: 2009 update by the Infectious Diseases Society of America. Clin Infect Dis 2009;49:1-45.

[56] Davidson JB, Edakkanambeth Varayil J, Okano A, Whitaker JA, Bonnes SL, Kelly DG, et al. Prevention of subsequent catheter-related bloodstream infection using catheter locks in high-risk patients receiving home parenteral nutrition. JPEN J Parenter Enteral Nutr 2017:41:685-90.

[57] Tribler S, Brandt CF, Petersen AH, Petersen JH, Fuglsang KA, Staun M, et al. Taurolidine-citrate-heparin lock reduces catheter-related bloodstream infections in intestinal failure patients dependent on home parenteral support: a randomized, placebo-controlled trial. Am J Clin Nutr 2017;106:839-48.

[58] Winnicki W, Herkner H, Lorenz M, Handisurya A, Kikić Ž, Bielesz B, et al. Taurolidine-based catheter lock regimen significantly reduces overall costs, infection, and dysfunction rates of tunneled hemodialysis catheters. Kidney Int 2018;93:753-60

[59] Arnoriaga Rodríguez M, Pérez de Ciriza Cordeu M, Camblor Álvarez M, Bretón Lesmes I, Motilla de la Cámara M, Velasco Gimeno C, et al. Clinical and economic impact of the taurolidine lock on home parenteral nutrition. Nutr Hosp 2018;35:761-6

[60] Bikle DD. Vitamin D insufficiency/deficiency in gastrointestinal disorders. J Bone Miner Res 2007:22:V50-4.

[61] Pittas AG, Laskowski U, Kos L, Saltzman E. Role of vitamin D in adults requiring nutrition support. JPEN J Parenter Enteral Nutr 2010;34:70-8.

[62] Nygaard L, Skallerup A, Olesen SS, Køhler M, Vinter-Jensen L, Kruse C, et al. Osteoporosis in patients with intestinal insufficiency and intestinal failure: prevalence and clinical risk factors. Clin Nutr 2018;37:1654-60.

[63] Cohen-Solal M, Baudoin C, Joly F, Vahedi K, D'Aoust L, De Vernejoul MC, et al. Osteoporosis in patients on long-term home parenteral nutrition: a longitudinal study. J Bone Miner Res 2003;18:1989-94.

[64] Pironi L, Arends J, Bozzetti F, Cuerda C, Gillanders L, Jeppesen PB, et al. ESPEN guidelines on chronic intestinal failure in adults. Clin Nutr 2016;35:247-307.

[65] Szczepanek K, Pedziwiatr M, Klek S. Denosumab improves bone mineral density in patients with intestinal failure receiving home parenteral nutrition: results from a randomized, controlled clinical trial. JPEN J Parenter Enteral Nutr 2018;42:652-7.
[66] Khaodhiar L, Keane-Ellison M, Tawa NE, Thibault A, Burke PA, Bistrian BR. Iron deficiency anemia in patients receiving home total parenteral nutrition. JPEN J Parenter Enteral Nutr 2002;26:114-9.

[67] Hwa YL, Rashtak S, Kelly DG, Murray JA. Iron deficiency in long-term parenteral nutrition therapy. JPEN J Parenter Enteral Nutr 2016;40:869-76.

[68] Santos D, Batoreu C, Mateus L, Marreilha Dos Santos AP, Aschner M. Manganese in human parenteral nutrition: considerations for toxicity and biomonitoring. Neurotoxicology 2014;43:36-45.

[69] Davila J, Konrad D. Metabolic complications of home parenteral nutrition. Nutr Clin Pract 2017;32:753-68.

[70] Hardy IJ, Gillanders L, Hardy G. Is manganese an essential supplement for parenteral nutrition? Curr Opin Clin Nutr Metab Care 2008;11:289-96.

[71] Pierce NF, Banwell JG, Rupak DM, Mitra RC, Caranasos GJ, Keimowitz RI, et al. Effect of intragastric glucose-electrolyte infusion upon water and electrolyte balance in Asiatic cholera. Gastroenterology 1968;55:333-43.

[72] Ko TC, Beauchamp RD, Townsend CM, Thompson JC. Glutamine is essential for epidermal growth factor-stimulated intestinal cell proliferation. Surgery 1993:114:147-53.

[73] Beaugerie L, Carbonnel F, Hecketsweiler B, Déchelotte P, Gendre JP, Cosnes J. Effects of an isotonic oral rehydration solution, enriched with glutamine, on fluid and sodium absorption in patients with a short-bowel. Aliment Pharmacol Ther 1997:11:741-6.

[74] Matarese LE, Abu-Elmagd K. Somatropin for the treatment of short bowel syndrome in adults. Expert Opin Pharmacother 2005;6:1741-50.

[75] Scolapio JS, Camilleri M, Fleming CR, Oenning LV, Burton DD, Sebo TJ, et al. Effect of growth hormone, glutamine, and diet on adaptation in short-bowel syndrome: a randomized, controlled study. Gastroenterology 1997;113:1074-81.

[76] Jeppesen PB, Pertkiewicz M, Messing B, Iyer K, Seidner DL, O’keefe SJ, et al. Teduglutide reduces need for parenteral support among patients with short bowel syndrome with intestinal failure. Gastroenterology 2012;143:1473-81.

[77] Schoeler M, Klag T, Wendler J, Bernhard S, Adolph M, Kirschniak A, et al. GLP -2 analog teduglutide significantly reduces need for parenteral nutrition and stool frequency in a real-life setting. Therap Adv Gastroenterol 2018:11, eCollection 2018.

[78] Pevny S, Maasberg S, Rieger A, Karber M, Blüthner E, Knappe-Drzikova B, et al. Experience with teduglutide treatment for short bowel syndrome in clinical practice. Clin Nutr 2019;38:1745-55.

[79] Jeppesen PB, Pertkiewicz M, Forbes A, Pironi L, Gabe SM, Joly F, et al. Quality of life in patients with short bowel syndrome treated with the new glucagonlike peptide- 2 analogue teduglutide-analyses from a randomised, placebocontrolled study. Clin Nutr 2013;32:713-21.

[80] Cserni T, Biszku B, Guthy I, Dicso F, Szaloki L, Folaranmi S, et al. The first clinical application of the spiral intestinal lengthening and tailoring (silt) in extreme short bowel syndrome. J Gastrointest Surg 2014;18:1852-7.

[81] Yannam GR, Sudan DL, Grant W, Botha J, Langnas A, Thompson JS. Intestinal lengthening in adult patients with short bowel syndrome. J Gastrointest Surg 2010;14:1931-6.

[82] Hommel MJ, van Baren R, Haveman JW. Surgical management and autologous intestinal reconstruction in short bowel syndrome. Best Pract Res Clin Gastroenterol 2016;30:263-80.

[83] Kong W, Wang J, Ying R, Li Y, Jin H, Mao Q, et al. A potential anatomic subtype of short bowel syndrome: a matched case-control study. BMC Gastroentero 2016;16:12. Epub 2016.

[84] Messing B, Lémann M, Landais P, Gouttebel MC, Gérard-Boncompain M, Saudin F, et al. Prognosis of patients with nonmalignant chronic intestinal failure receiving long-term home parenteral nutrition. Gastroenterology 1995;108:1005-10.

[85] Pironi L, Paganelli F, Labate AM, Merli C, Guidetti C, Spinucci G, et al. Safety and efficacy of home parenteral nutrition for chronic intestinal failure: a 16-year experience at a single centre. Dig Liver Dis 2003:35:314-24.

[86] Grant D, Abu-Elmagd K, Mazariegos G, Vianna R, Langnas A, Mangus R, et al. Intestinal transplant registry report: global activity and trends. Am J Transplant 2015;15:210-9.

[87] Marino IR, Lauro A. Surgeon's perspective on short bowel syndrome: where are we? World J Transplant 2018;8:198-202.

[88] Matsumoto CS, Subramanian S, Fishbein TM. Adult intestinal transplantation. Gastroenterol Clin North Am 2018;47:341-54. 